\title{
COST ANALYSIS OF EMERGENCY VISITS DUE TO DRUG RELATED PROBLEMS
}

\author{
ELSA MATHEW ${ }^{1 *}$, C. SAKTHI THAVA PRIYA ${ }^{1}$, S. AKASH ADITYA ${ }^{1}$, G. SASIKALA ${ }^{1}$, ASHLY ABRAHAM ${ }^{1}$, \\ RAGHUPATHI V. ${ }^{2}$
}

\author{
1Department of Pharmacy Practice, PSG College of Pharmacy, Coimbatore, Tamilnadu, India, ${ }^{2}$ Emergency Department, PSG Hospitals, \\ Coimbatore, Tamilnadu, India \\ Email: elzamathew27@gmail.com
}

Received: 09 May 2019 Revised and Accepted: 04 Jul 2019

\begin{abstract}
Objective: To identify patients coming to Emergency Medicine Department (EMD) with drug related problems, classify the DRPs and calculate the direct cost spent for treating them.

Methods: This was a prospective observational study conducted in emergency medicine department. The patients coming to EMD with DRPs were classified according to Cipolle's classification and the direct medical and non-medical costs were calculated.

Results: A total of around 107 patients identified with DRPs of which 99 patients were included in the study. In this study, $51 \%$ of the cases were due to ADR and 35\% due to non-adherence and rest of the cases were due to overdose (10\%), drug interaction (3\%) and sub therapeutic dose (1\%). Major portion for treatment was spent for direct medical cost in which cost for laboratory investigations have contributed the most, INR 10,93,992 (42\%) followed by Health care professional cost INR 55,6814 (21\%), Pharmacy cost INR 4,00,524.6 (15\%), Admission cost INR 3,80,400 (15\%). The direct non-medical cost includes cost for diet and travel which was found to be INR 1,68,443 and INR 71,947 respectively.
\end{abstract}

Conclusion: The drug related problems adds a significant economic burden on the patients which can be reduced by imparting knowledge about the proper use of medicines and by improving collaborative efforts of the patients, physicians, pharmacists and caregivers.

Keywords: Drug related problems (DRPs), Cost analysis, Direct cost

(C) 2019 The Authors. Published by Innovare Academic Sciences Pvt Ltd. This is an open access article under the CC BY license (http://creativecommons.org/licenses/by/4.0/) DOI: http://dx.doi.org/10.22159/ijpps.2019v11i8.34016

\section{INTRODUCTION}

Medications are used in various disease conditions to optimize drug therapy with minimum safety-related problems within the framework of the pharmaceutical care plan. Over the last few decades, advances in drug, therapies have improved patient care and there has been an apparent increase in the incidence of DrugRelated Problems (DRPs) being reported [1]. DRP can be defined as an event which may potentially affect the health outcomes in the patients. It can occur during any stages of drug use, starting from prescribing to administration. Lack of follow-ups may also leads to drug-related problems [2]. Various risk factors for DRPs include polypharmacy, comorbid diseases, increased medicine use. Another major contributing factor is the practice of self-medication with over the counter (OTC) medications available in India and many other parts of the world [3-5].

Even with the combined efforts taken by the manufacturers, regulatory authorities and health care professionals to reduce the incidence of DRPs, they continue to occur due to various reasons that can be attributed to different stages of drug use. DRPs occur in patients because of the improper use that might appear at any level of drug use. This demands appropriate attention and interventions to reverse the condition which might expose a patient to severe harm than the disease itself for which one was taking medicine. The severity of DRPs can range from minor side effects to the death of the patient.

Nowadays, a lot of hospital visits and admissions can be attributed to DRPs and this rate seems to be rising over the past two decades. In the process of reversing the DRPs healthcare resources and a significant amount of money is spent, which poses an economic burden on the patients as they have to spend more than what was intended for their ailment. The economic impact of DRPs is extensive on patients, healthcare providers, administrators, and society. Estimates have shown that for every US $\$ 1$ spent on drugs, US $\$ 1.33$ is consumed to treat DRPs [6]. The cost of DRP has increased every year and in 2008 it was estimated at US $\$ 289$ billion in the United States [7]. An Indian study showed total cost for the management of
DRPs was calculated to be INR17,37,339. The direct cost was INR $1,72,961$ and the approximate indirect cost was INR 15,64,378. The drugs used for treatment (54\%) contribute a significant part in direct cost. Among various drug-related problems, medication nonadherence was the main contributor ( $85 \%$ of total cost) followed by adverse drug reactions (13\% of total cost) [8].

In a developing country like ours, the majority of the population belongs to the middle class with only sufficient money for their day to day living and do not have medical insurance coverage. Also, most of the medications are available as OTC drugs and lack of qualified pharmacy professionals for handling the medicine. Therefore, creating a clear picture of hospital visits due to drug-related problems and its economic impact is significant. Even though a number of studies have evaluated the pharmacoeconomic impact of DRPs in the developed world, only limited data are available from developing countries. In our study, we have attempted to calculate the direct cost involved to treat patients who present to the Emergency Department because of DRPs.

\section{MATERIALS AND METHODS}

A prospective observational study was conducted over a period of six months in the Emergency medicine Department of PSG Hospitals, Peelamedu, Coimbatore. The study was approved by the Institution Human Ethics Committee (IHEC, PSG IMSR) of the hospital. The protocol was approved on $26 / 2 / 2018$, Proposal number: $18 / 077$. Patients came to emergency medicine department with drug-related problems were selected for the study. Both genders and all age groups were included. Critically ill patients, patients who are not willing to participate and patient's with claim or insurance were excluded from the study.

Patient data collection form included demographic details, medical and medication history, direct medical and non-medical cost. The DRPs identified and were categorized based on CIPPOLLEs classification, which include Adverse drug reactions (ADR), Medication error, Noncompliance, Drug interactions, Untreated 
indication, Drug use without indication, Improper drug selection, Subtherapeutic Dose, Supra therapeutic Dose. The ADR's were classified based on the WHO-UMC scale and preventability of ADR was categorized based on the Schumock and Thornton scale. The direct cost for each DRPs was calculated. Pharmacy cost, Laboratory cost, Admission cost, Health care professional cost were included in the direct medical cost, whereas direct non-medical cost included Diet and Transport charges.

\section{RESULTS}

During our study period of 6 mo, 10,485 patients visited the emergency department, of which 107 were due to drug-related problems. From these cases, 8 were excluded after meeting the exclusion criteria and the remaining 99 cases were included in our study.
The DRPs were found to be almost equally predominant in both genders, of which $48 \%$ were females and $52 \%$ were males. The 99 subjects enrolled in our study were classified into 5 age groups ranging from 0 $18,18-29,40-59,59-79$ and 80-99 y. Most frequent occurrence of DRP was observed in the age group $40-59$, which was about $45 \%$.

The DRP's observed were grouped according to CIPOLLE's classification into 5 categories. The patients included in our study experienced different types of drug related problems like adverse drug reactions, non-compliance or medication error, drug interactions, sub-therapeutic dose and overdose. In this study, 50 patients were presented with ADR (51\%), followed by 35 nonadherent to the medicines. Overdose of medications were observed in 10 patients $(10 \%)$, while in 3 patients drug interactions were seen $(3 \%)$ and and a case of sub therapeutic dose was recieved which is shown in fig. 1 .

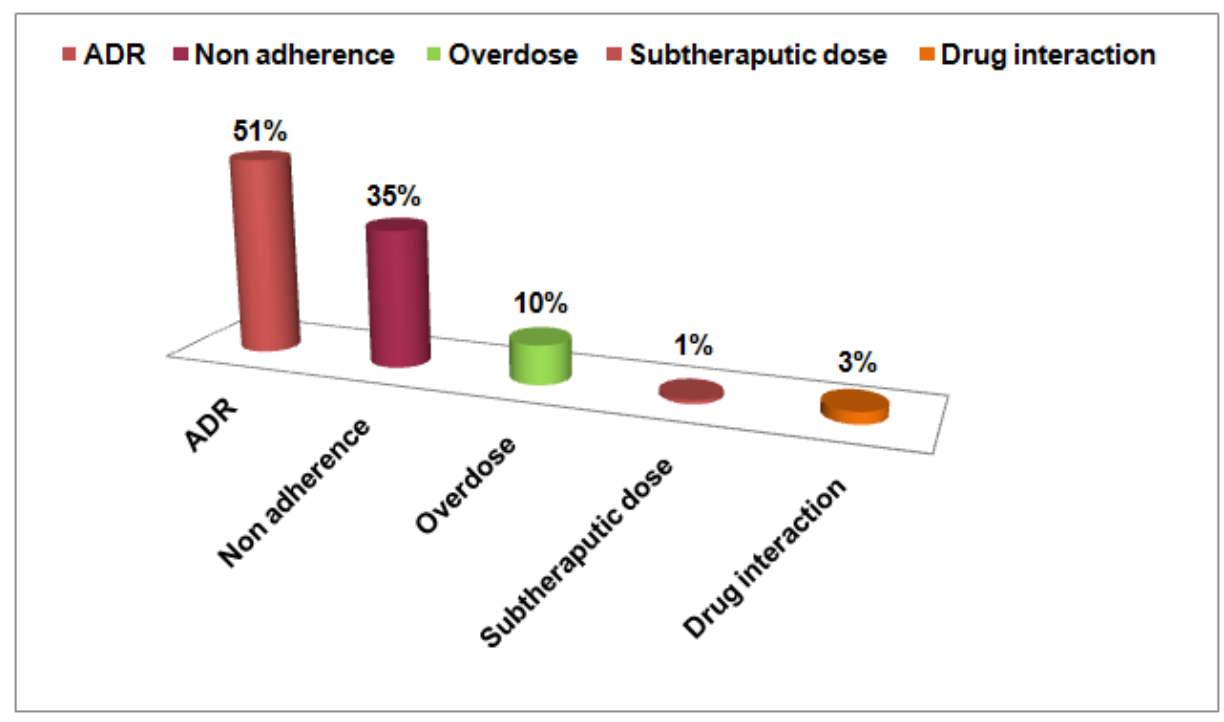

Fig. 1: Distribution of DRP's as per cipolle's classification

In the DRPs, ADR showed the highest prevalence and was seen in 50 patients (51\%). The ADR's were classified based on the WHO-UMC scale and it showed $64 \%$ was probable, $32 \%$ possible and $4 \%$ certain. The preventability of ADR was categorized based on the Schumock and Thornton scale, which revealed 34\% of ADR's were not preventable and $66 \%$ were preventable of which $30 \%$ were definitely preventable which is shown in the fig. 2 and 3 . This may be due to the selection of drug, dose, route of administration, frequency, drug interaction, lack of patient's knowledge. Of the 99 cases assessed, 15 subjects were admitted due to DRPs associated with anti-diabetics (14\%) followed by 14 admissions by antiepileptics (13\%) and anti-psychotics (13\%) each.

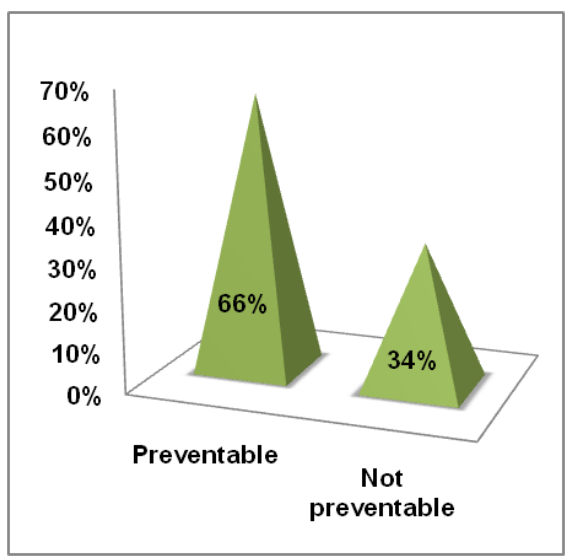

Fig. 2: Preventability of ADR

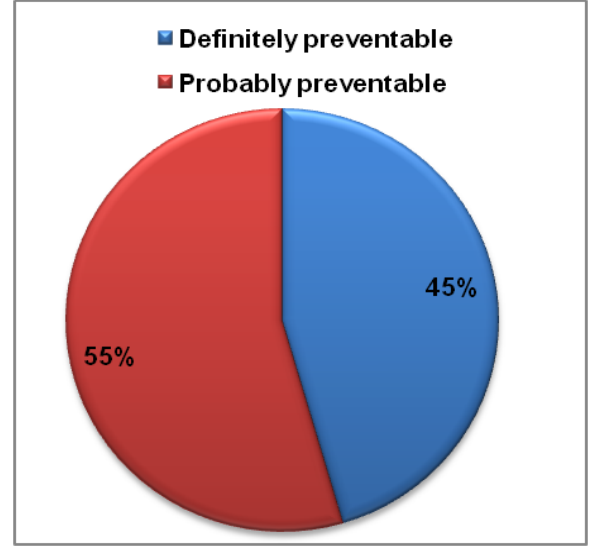

Fig. 3: Preventability class,

The direct cost spent in treating DRP's is an addition of direct medical cost and direct non-medical cost. Direct medical cost includes pharmacy cost, healthcare professionals cost, laboratory cost, admission cost that contributed to almost $92 \%$ of the total and direct non-medical cost included diet and travel charges. A total direct cost of about INR 25,96,007 was spent to treat the patients who visit the EMD due to drug-related problems. The patients were treated in both inpatient (IP) and outpatient (OP) settings. For managing the DRPs for outpatients, INR 75,248 (33\% of the total direct cost) was spent and for those treated as inpatients, an amount of INR $25,20,829$ ( $67 \%$ of the total direct cost) was disbursed. A total 
of INR 23,56,728 was spent for direct medical cost of which, $42 \%$ comprised of laboratory charges amounting to INR 10,39,992. A direct non-medical cost of INR 2,39,349 was spent by the patients during our study period.6\% of the total direct cost was the amount spend towards diet which is the highest contributor in direct nonmedical cost of INR 1,68,443 were shown in table 1 .

Table 1: Distribution of DRPs in treatment cost

\begin{tabular}{|c|c|c|c|c|}
\hline Type of cost & Total cost (in INR) & Percentage (\%) & Mean & Standard deviation ( \pm ) \\
\hline \multicolumn{5}{|l|}{ Direct medical cost } \\
\hline Pharmacy cost & 400524.6 & $15 \%$ & 4045.70 & 17849.91 \\
\hline Health care professionals cost & 556814 & $21 \%$ & 5624.38 & 14280.99 \\
\hline Laboratory cost & 1093992 & $42 \%$ & 11050.42 & 19525.63 \\
\hline Admission cost & 380400 & $14 \%$ & 3842.42 & 11268.86 \\
\hline Total & 2356728.148 & $92 \%$ & 23805.33 & 47071.31 \\
\hline \multicolumn{5}{|l|}{ Direct non-medical cost } \\
\hline Diet & 168443 & $6 \%$ & 1701.44 & 2319.83 \\
\hline Travel & 71947 & $2 \%$ & 726.73 & 768.90 \\
\hline Total & 239349.2 & $8 \%$ & 2417.66 & 2797.86 \\
\hline Grand total & 2596077 & $100 \%$ & 26223.00 & 48568.96 \\
\hline
\end{tabular}

In the total direct cost, $67 \%$ was contributed by 66 in-patients and $33 \%$ by the out-patients. Even though the direct cost for inpatients and out-patients was found to be similar, direct nonmedical cost (25\%) was found to be a major contributing factor for out-patients whereas direct medical cost was substantial for in-patients.

In our study the direct cost spent for ADR was INR 15,20,960 (58\%), which shows ADR is the highest contributor among the DRPs, then comes non adherence with INR 8,32,656 (32\%)and for druginteraction and overdose it was INR 1,93,888 (6\%) and INR 1,23,052 (4\%) respectively.

The direct cost spent for ADR was about INR 15,20,960, in which direct medical cost was INR 13,94,037 and the direct non-medical cost was INR 1,26,923. Among various drug-related problem's adverse drug reactions (ADR) was the main contributor $(51 \%)$ of total cost, it was observed that the potential for an adverse reaction like cutaneous reaction like rash, generalized pruritus, was $43 \%$ followed by hepatotoxicity. ATT had the highest potential (44\%) for an adverse drug reaction followed by steroids (42\%).

Non-adherence to anti-diabetic, anti-epileptics, anti-tubercular, antihypertensive drugs was predominant DRPs reported, which escalates the treatment cost especially the direct medical cost Approximately about INR 23790.17 was spent by a patient, in which direct medical cost was INR 21501.95 and the direct non-medical cost was INR 2288.22.

The direct cost spent for drug-interaction was about INR 1,93,888, in which direct medical cost was INR 1,77,864 and the direct nonmedical cost was INR 16,024 . The laboratory cost shows the highest percentage was about 53\% from the total medical cost. Management of drug interaction leading to hospitalization incurred the least expenditure was about $3 \%$.

Among the 10 overdose cases, the major portion of the total cost was spent for healthcare professionals (34\%), which mainly includes the fees for physicians, nurses, and physiotherapist and is about INR 4238. The direct cost spent for overdose was about INR1,23,0522 in which direct medical cost was INR 1,11,60 and the direct nonmedical cost was INR 11,4480

The direct cost spent for sub-therapeutic dose was about INR 7972.49 in which direct medical cost was INR 7272.49 and the direct non-medical cost was INR 700.

\section{DISCUSSION}

The prevalence of DRPs was higher in males (52\%) than in females $(48 \%)$ which were also comparable to the population in an Indian study [8]. About $45 \%$ of DRP was observed in the age group 40-59 which may be due to poor knowledge about the disease and the drugs and other risk factors such as poly-pharmacy, co-morbid conditions and lack of follow-up and reassessment of medica treatment. Similarly, in a study by Baena MI et al., the patients within the age group of 45-64 taking 3 or more medicines presented an odds ratio of 64.07 [9]

Among the various DRPs, ADRs showed the highest prevalence. A study by Peter J Zed et al. showed drug-related visits were predominantly due to adverse drug reactions, followed by nonadherence which was almost identical to our findings [10]; while overprescribing of the correct medication was found to be the major cause in the study by Tafreshi et al. [11]. The distribution of ADRs based on preventability showed that most of the ADRs were preventable. Adverse drug-related events accounted for $12 \%$ of emergency department visits, of which $68 \%$ were considered preventable in the first study [10]. Apart from these, a study from Karnataka showed that $47.55 \%$ of DRPs are due to drug interaction [12].

In our study most common drug classes that caused DRPs were antidiabetics, anti-psychotics, anti-epileptics. Peter J Zed et al., in one of his study identified antimicrobial agents (11.2\%), opioid-containing analgesics (11.2\%), antipsychotics $(9.5 \%)$ and benzodiazepines $(6.1 \%)$ as the major contributors of DRPs [10]. In his other study in 2018 the most common drug classes were anti-infectives $(27.4 \%)$, respiratory agents $(22.4 \%)$, central nervous system agents $(20.4 \%)$, immunosuppressants (7.5\%), and gastrointestinal agents $(6.0 \%)$ [13]. A study by Tafreshi revealed the most common medications involved were cardiovascular agents, narcotics, antibiotics, asthma medications, antidepressants, anti-epileptics, acetaminophen, antiParkinson medications [11].

In treating DRPs a significant amount of money is spent and it is actually an additional expense to the normal cost of drug use by the patients. A study conducted in India by Smita Pattanaik et al., showed that the total cost of management of all the 92 drug-related events was calculated to be INR 17,37,339. The major share of the direct cost was due to the drugs used for the treatment of DRPs (54\%). In addition, medication noncompliance was the single most important contributor to the cost ( $85 \%$ of the total cost), followed by ADRs $(13 \%$ of the total cost), while management of drug overdose leading to hospitalization incurred the least expenditure [8].

When a person visits the hospital due to a DRP, if treated in an inpatient setting, an average of about INR 38,194.38 is spent and if treated as an outpatient, an average of about INR 2,280.243 is spent. The patients, based on their DRP's severity and the amount of attention needed to reverse the condition, they are decided whether they have to be treated in the outpatient setting or whether they have to be treated in the inpatient setting and accordingly the cost for the reversal of DRPs vary as mentioned above. Usually, when the patient is severely affected, he/she might have to be admitted in the hospital in the care of various healthcare professionals to reverse the DRP or if the DRP is not that severe, like in allergies caused by the drugs, the patient is stabilized and then discharged without admission in the hospital. 


\section{CONCLUSION}

A large portion of DRPs observed were preventable cases of ADRs and non-adherence to treatment. The most common drug classes involved were anti-diabetics, anti-epileptics, anti-psychotics and anti-hypertensive drugs which are used for the predominant diseases in India. Laboratory cost was found to be the major contributor for direct medical cost. Educating patients about the correct use of their medications, showing the importance of adherence to treatment could reduce pressures on emergency services, hospitalizations, and consequently costs to the hospital, the health system, and the society.

\section{LIMITATIONS OF THE STUDY}

The main limitation of our study is that direct cost is only calculated. Indirect cost and intangible cost, which are the significant dimensions of cost of illness studies, were not included.

\section{AUTHORS CONTRIBUTIONS}

All the author have contributed equally

\section{CONFLICTS OF INTERESTS}

All authors have none to declare

\section{REFERENCES}

1. M Sonal Sekhar, C Adheena Mary, PG Anju, Nishana Ameer Hamsa. Study on drug-related hospital admissions in a tertiary care hospital in South India. Saudi Pharma J 2011;19:273-8.

2. Adusumilli PK, Adepu R. Drug-related problems: an overview of various classification systems. Asian J Pharm Clin Res 2014;7:7-10.

3. Sundus Basharat. Management of drug-related hospital admissions. Int J Sci Res Publications 2016;6:63-7.

4. Shankar PR, Partha P, Shenoy N. Self-medication and nondoctor prescription practices in pokhara valley, Western Nepal: a questionnaire-based study. BMC Fam Pract 2002;3:17.
5. Selvaraj K, Kumar SG, Ramalingam A. Prevalence of selfmedication practices and its associated factors in Urban Puducherry, India. Perspect Clin Res 2014;5:32-6.

6. Johnson JA, Bootman JL. Drug-relatedmorbidityandmortality: acost-of-illnessmodel. Arch Int Med 1995;155:1949-56.

7. Gabriel Rodrigues Martins de Freitas, Mariana Younes Tramontina, Giacomo Balbinotto, Dyfrig Arwyn Hughes, Isabela Heineck. Economic impact of emergency visits due to drugrelated morbidity on a Brazilian Hospital. Value Health Regional Issue 2017;14C:1-8.

8. Smita Pattanaik, Punit Dhamija, Samir Malhotra, Navneet Sharma, Promila Pandhi. Evaluation of cost of treatment of drug-related events in a public care tertiary sector. Br J Clin Pharmacol 2009;67:363-9.

9. Baena MI, Faus MJ, Fajardo PC, Luque FM, Sierra F, Martinez Olmos J, et al. Medicine-related problems resulting in emergency department visits. Eur J Clin Pharmacol 2006;62:387-93.

10. Peter J Zed, Riyad B Abu-Laban, Robert M Balen, Peter S Loewen, Corinne M Hohl MD, Jeffrey R Brubacher, et al. Incidence, severity and preventability of medication-related visits to the emergency department: a prospective study. Canadian Med Assoc Licensors 2008;178:1563-9.

11. Tafreshi MJ, Melby MJ, Kaback KR, Nord TC. Medication-related visits to the emergency department: a prospective study. Ann Pharmacother 1999;33:1252-7.

12. Sarfaraz Mohammed, Sanjay Poudel, Fernance Laloo, Arjun Madhur, Rinson Robert, Binu Mathew. Assessment of drugrelated problems in a tertiary care teaching hospital, india. Asian J Pharm Clin Res 2017;10:310-3.

13. Peter J Zed, Karen JL Black, Eleanor A Fitzpatrick, Stacy Ackroyd Stolarz, Nancy G Murphy, Janet A Curran, et al. Medication-related emergency department visits in pediatrics: a prospective observational study. Pediatrics 2018;135:435-43. 